\title{
Correlative analysis in Geosciences: where are we at? where are we going?
} RiCHARD J M TAYLOR ${ }^{1}$, EDDY HILL ${ }^{1}$, RUMANA KHAN ${ }^{1}$,
TIM E JOHNSON $^{2}$, CHRIS ClARK $^{2}$, PIERRE LANARI $^{3}$

${ }^{1}$ Carl Zeiss Microscopy Ltd, ZEISS Group, Cambourne, Cambridge UK

${ }^{2}$ School of Earth and Planetary Sciences, Curtin University, Perth, Western Australia

${ }^{3}$ Institute of Geological Sciences, University of Bern, Bern, Switzerland

(richard.taylor@zeiss.com)

Geoscientists use a wide variety of tools covering applications from imaging to microanalysis. As our collective fields have advanced the information provided by each technique has become increasingly rich with greater resolution, increasing precision, more analyses, more elements and ever expanding metadata. Coupled with this is a more esoteric problem, even when faced with an identical instrument, geoscientists often incorporate bespoke workflows, with unique element lists, and task specific image resolutions.

The example of geochronology in metamorphic rocks demonstrates the complexity of the task ahead, with workflows progressing through many of the common Geoscience techniques. Light microscopy images are the start of most petrological research, but are hard to digitise in a meaningful way. Electron beam techniques can combine petrological and textural observations with geochemical information at the thin section scale. Electron and ion beam in situ analysis provides additional trace element and isotopic information but for comparatively small volumes and in multiple formats. An additional challenge is how to link this information to model outputs, such as the pressure and temperature information obtained from thin section analysis.

A consistent and robust approach to collecting and archiving data opens up further opportunities to streamline our research. Projects associated with 'big data' lend themselves to automated approaches with efficiency supported by machine learning and cloud based workflows.

The digital revolution gives us the opportunity to tackle these new challenges. As we innevitably progress towards greater integration of very diffreent techniques, we must develop mechanisms to correlate these disparate datasets. This is essential not just for personal research, but sharing datasets with colleagues and collaborators, efficient supervison of research students, and ensuring quality assessment of published works. 